\title{
NcRNAs in Vascular and Valvular Intercellular Communication
}

\author{
Benedikt Bartsch ${ }^{1 \dagger}$, Philip Roger Goody ${ }^{1 \dagger}$, Mohammed Rabiul Hosen ${ }^{1}$, Denise Nehl ${ }^{1}$, \\ Neda Mohammadi ${ }^{2}$, Andreas Zietzer ${ }^{1}$, Philip Düsing ${ }^{1}$, Alexander Pfeifer ${ }^{2}$, Georg Nickenig ${ }^{1}$ \\ and Felix Jansen ${ }^{1 *}$ \\ ${ }^{1}$ Department of Internal Medicine II, Heart Center Bonn, University Hospital Bonn, Bonn, Germany, ${ }^{2}$ Institute of Pharmacology \\ and Toxicology, University Hospital Bonn, Bonn, Germany
}

Non-coding RNAs have been shown to be important biomarkers and mediators of many different disease entities, including cardiovascular (CV) diseases like atherosclerosis, aneurysms, and valvulopathies. Growing evidence suggests a central role of ncRNAs as regulators of different pathological pathways involved in endothelial dysfunction, cardiovascular inflammation, cell differentiation, and calcification. This review will

OPEN ACCESS

Edited by:

David De Gonzalo-Calvo, Lleida Institute for Biomedical Research (IRBLleida), Spain

Reviewed by:

Dennis Mehrkens,

University of Cologne, Germany Nan Li,

Ludwig Maximilian University of Munich, Germany

*Correspondence:

Felix Jansen

Felix.jansen@ukbonn.de

${ }^{\text {t}}$ These authors have contributed equally to this work

Specialty section: This article was submitted to

Protein and RNA Networks, a section of the journal Frontiers in Molecular Biosciences

Received: 29 July 2021 Accepted: 23 September 2021 Published: 05 November 2021

Citation:

Bartsch B, Goody PR, Hosen MR, Nehl D, Mohammadi N, Zietzer A,

Düsing $P$, Pfeifer $A$, Nickenig $G$ and Jansen $F$ (2021) NcRNAs in Vascular and Valvular

Intercellular Communication. Front. Mol. Biosci. 8:749681. doi: 10.3389/fmolb.2021.749681 discuss the role of protein-bound and extracellular vesicular-bound ncRNAs as biomarkers of vascular and valvular diseases, their role as intercellular communicators, and regulators of disease pathways and also highlights possible treatment strategies.

Keywords: ncRNA, atherosclerosis, aortic stenosis, endothelial dysfunction, ncRNA therapy

\section{INTRODUCTION}

Non-coding RNAs (ncRNAs) consists of transfer RNA (tRNA), microRNA (miRNA, or miR), long noncoding RNA (lncRNA), circular RNA (circRNA), and other small RNAs. NcRNA expression has been shown to correlate with several cardiovascular diseases including aortic stenosis. Modulating ncRNA expression in vitro has also been shown to affect disease progression (Das, 2020). While considerable advances in understanding the molecular functions of ncRNAs in vitro have been achieved in the last years, unravelling the role of ncRNAs in vivo, their establishment as biomarkers and possible use as potential therapeutics are still in its infancy. Therefore, ncRNAs are promising targets for further research.

\section{REGULATION OF CELLULAR NCRNA EXPRESSION UNDER PHYSIOLOGICAL AND PATHOLOGICAL CONDITIONS}

NcRNA expression in CV cells can vary, depending on the pathophysiological condition of the parent cell. Different stimuli, such as glucose levels, oxidative stress, inflammation, and an osteogenic milieu-all important in CV pathologies-can influence ncRNA expression in the affected cells (Libby et al., 2019; Yuan et al., 2019). Current knowledge on synthesis and maturation of different classes of ncRNAs, under physiological and pathological conditions, will be summarized and discussed in this section.

\section{Hyperglycemia}

High plasma glucose concentrations are a major risk factor for atherosclerosis as well as aortic stenosis (Cosentino, 2019; Goody et al., 2020). In vitro, human umbilical vascular endothelial cells (HUVECs) exposed to high glucose medium displayed an upregulation of 214 lncRNAs, while 197 
were downregulated and several ncRNAs interfered directly with glucose metabolism, while 945 possible lncRNA-mRNA pairs were found, indicating a strong regulatory link (Sun and Wong, 2016; Xu et al., 2020a).

In a diabetic mouse model, the lncRNA metastasis associated lung adenocarcinoma transcript 1 (MALAT1) mediated proinflammatory cytokine expression was altered according to glucose concentration and MALAT1 inhibition lead to a diminished inflammatory response as well as reduced endothelial cell apoptosis and tube formation in retinal cells (Radhakrishnan and Kowluru, 2021).

Plasmacytoma variant translocation 1 (PVT1), another apoptosis mediator, is upregulated in kidney cells exposed to high glucose levels and mediates hypoxic cardiac injury by acting as a sponge for miR-135a-5p, thus upregulating Forkhead box O1 (FOXO1)-mediated apoptosis (Sun and Wong, 2016; Xu et al., 2020b).

Recently, Liu et al. demonstrated a glucose-dependent steroid receptor RNA activator (SRA) mediated increase in insulin sensitivity, most likely via the insulin-like-growth-factor 1 (IGF1) and PPAR $\gamma$ signaling pathway (Liu et al., 2014a; Liu et al., 2014b; Liu et al., 2016). Unlike IGF1 and PPAR $\gamma$, SRA coactivation negatively regulates Toll-like-recptor 4 (Tlr4) and subsequent TNFa release, both of which have been linked to the pro-inflammatory response in the early stages of atherosclerosis and aortic stenosis (Chong et al., 2004; Xu et al., 2010; Liu et al., 2016; Goody et al., 2020).

The lncRNA myocardial infarction-associated transcript (MIAT) has initially been associated with myocardial infarction but can also act as a sponge for miR-150-5p, which regulates VEGF-expression, and its expression is increased in patients with renal dysfunction and high blood glucose levels (Yan et al., 2015; Sun and Wong, 2016).

\section{Oxidative Stress}

Another mediator of vascular and valvular damage on a cellular level is oxidative stress (Kattoor et al., 2017; Goody et al., 2020).

NcRNAs are differentially expressed in monocytes and macrophages isolated from human blood samples from patients with and without high risk for atherosclerosis (Liu et al., 2014c; Yan et al., 2015). Linc-TP53I13 and lincPOTED8 are overexpressed in an in vitro model mimicking oxidative stress through lipopolysaccharide exposure in monocytes and adipocytes and in obese patients (Liu et al., 2014c). MIAT1 expression is increased in cells exposed to oxidative stress (Yan et al., 2015).

\section{Inflammation}

TNFa is major signaling molecule in innate and adaptive immunity responses in different tissues (Whitley et al., 1994). One of its many pro-inflammatory downstream signaling pathways includes the NF- $\kappa \mathrm{B}$ pathway, which induces gene expression of cytokines such as IL-1, and different miRs as well as IncRNA (e.g., LincRNA-Cox2). The regulated ncRNAs have been shown to often lie adjacent to coding genes that were also regulated by NF- $\kappa$ B such as Cox2 Divergent and Gp96 Convergent (Rapicavoli et al., 2013).
LincRNA-Cox2 is found proximally to the prostaglandinendoperoxide synthase 2 (Cox2) gene locus and its expression is promoted in a pro-inflammatory environment, such as after TLR-2 and -4 stimulation or after LPS stimulation in macrophages in vitro (Guttman et al., 2009; Carpenter et al., 2013). The activation of TLRs plays a key role in atherosclerosis and myocardial infarction (Chong et al., 2004). LincRNA-Cox2 downregulates the expression of immune genes, similar to an auto-feedback-mechanism, by binding to heterogeneous nuclear ribonucleoproteins (hnRNPs) in order to repress transcription (Carpenter et al., 2013).

While ncRNAs were shown to regulate inflammatory responses in a variety of cardiovascular diseases, the number of studies investigating the role of ncRNAs in aortic stenosis remains low. Yet, key promoters of aortic stenosis such as TNFa, members of the Wnt-pathway, and TLR activation are modulated by ncRNA expression and thus may provide a promising target for future investigations (see Figure 1) (Wang et al., 2013; Venardos et al., 2014; Goody et al., 2020). Recently published data suggests a key role of ncRNA in regulating oxLDL-uptake, endothelial-to-mesenchymal-transformation (EndMT) and valvular calcification (Mahmut et al., 2014; Rayner, 2020).

\section{Osteogenic Milieu}

NcRNA expression is also modified in osteogenic milieus and can promote osteoblastic differentiation of cardiovascular cells, a major driver of calcific valve disease and atherosclerotic plaque development (Alexopoulos and Raggi, 2009; Goody et al., 2020). MiR-30 family members moderate mesenchymal stem cell (MSC) transformation to osteocytes by regulating Runx2expression (Eguchi et al., 2013). In human aortic valvular interstitial cells that have been treated with an osteogenic medium, miR-30 b/c are downregulated during MSC transformation, while miR-30 a, d, and e are upregulated with miR-30e acting as a co-activator of the Wnt-pathway and inductor of Runx2 expression in human coronary artery smooth muscle cells (HCASMC) (Nigam et al., 2010; Eguchi et al., 2013; Wang et al., 2013). MiR-125b, miR-143 and -145 are downregulated in vitro in human vascular smooth muscle cells (HVSCM) after treatment with high levels of inorganic phosphate to promote osteogenic differentiation in these cells (Coffey and Jones, 2014). Furthermore, miR-125b was found to be downregulated in an in vivo model of atherosclerosis and aortic calcification in mice (Goettsch et al., 2011; Rangrez et al., 2012; Coffey and Jones, 2014), while miR-223 was overexpressed in calcified murine aortas (Rangrez et al., 2012). Human calcific aortic valve disease is associated with significantly reduced miR-204 levels and miR-204 mimics suppressed the osteogenic activity of interstitial cells from diseased valves (Song et al., 2020).

Furthermore, MALAT1 has been demonstrated to promote osteogenic differentiation in an osteogenic milieu via an increased ALP activity and Runx2 activation (Wang et al., 2020). MALAT1 is regulated in an osteogenic milieu via human antigen $\mathrm{R}$ ( $\mathrm{HuR}$ ) expression, which itself is upregulated by MALAT1 via inhibition of miR-191-3p, establishing a positive feedback loop for osteogenic differentiation (Wang et al., 2020). 


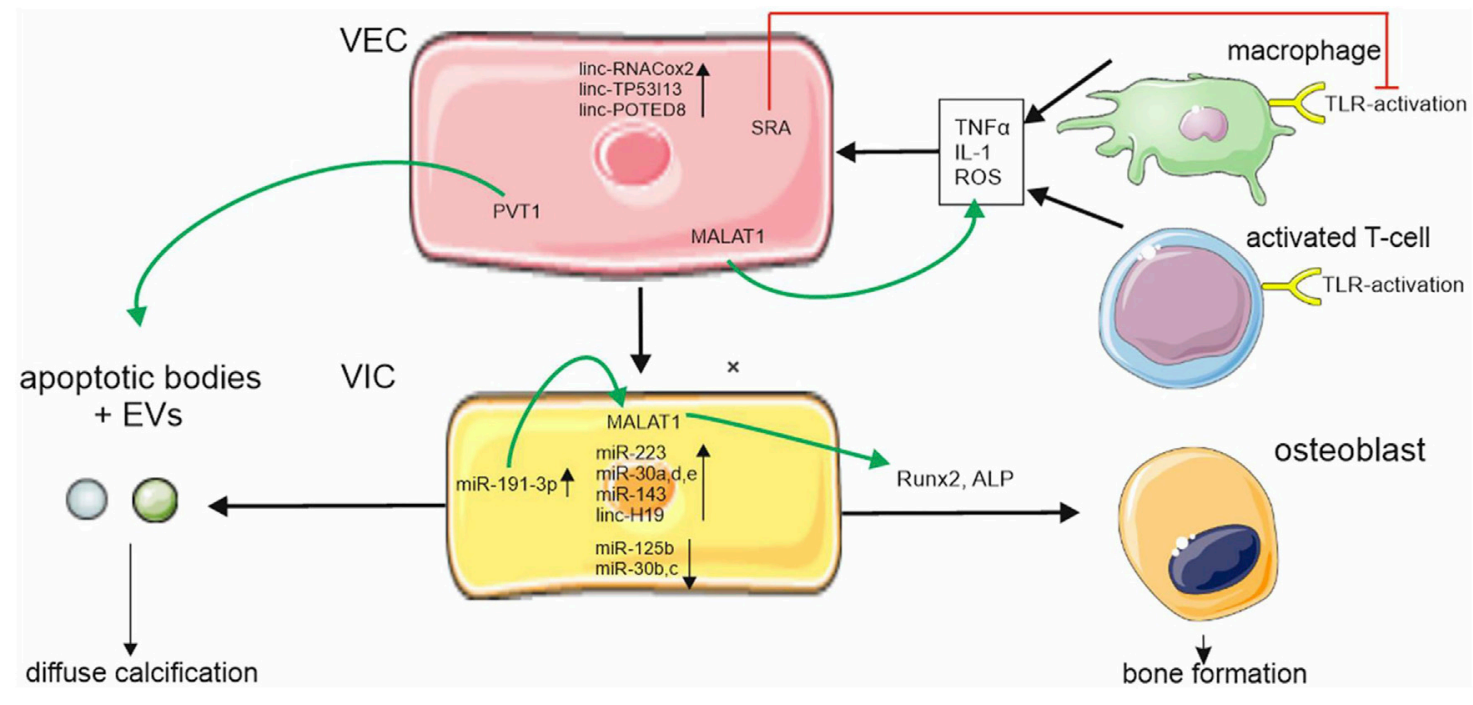

FIGURE 1 | ncRNA regulation in aortic stenosis. ncRNAs are differently expressed during aortic stenosis disease progression in valvular endothelial cells (VEC) and valvular interstitial cells (VIC). Metastasis associated lung adenocarcinoma transcript 1 (MALAT1) is upregulated in VECs und VICs and increases pro-inflammatory cytokine expression and regulators of osteoblastic differentiation. Plasmacytoma variant translocation (PVT1) regulates apoptosis. Apoptosis is part of a complex system leading to programmed cell death thus causing increased calcium uptake of VICs through cell debris steroid receptor RNA activator (SRA) which downregulates Toll-like receptor (TLR) activation. Cellular symbols were adopted with permission from smart.servier.com and Vecteezy.

\section{CELLULAR SELECTION AND PACKAGING MECHANISMS OF NCRNAS INTO EXTRACELLULAR CARRIERS}

Growing evidence suggests that secreted ncRNA profile reflects the state of the parent cell and can be released directly into the extracellular space/blood stream, bound to RNA-binding proteins such as Argonaute 2 (Ago-2) or be (selectively) packaged into different forms of extracellular vesicles (EVs) (Kim et al., 2017). These EVs include exosomes, microvesicles and apoptotic bodies.

The ncRNA content of EVs often does not reflect their corresponding concentrations in the cytoplasm of the originating cell, thus underlining the concept that ncRNA sorting into EVs and secretion are actively regulated cellular processes that are relevant for intercellular communication (Villarroya-Beltri et al., 2013; Gezer et al., 2014; Shurtleff et al., 2016).

MiRNAs with gene sequences GGAG, C/UCCU/G, so called EXOmotifs, in the $3^{\prime}$ half of the RNA were found overrepresented in EVs, while miRNAs with mutated EXOmotifs were not detected in EVs, indicating a cause-effect relation between EXOmotifs and EV packaging, potentially mediated via heterogeneous nuclear ribonucleoproteins (HNRNP) (Villarroya-Beltri et al., 2013; Zietzer et al., 2020). EV sorting functions as a tool for cellular ncRNA homeostasis. Thus, ncRNA EV levels are reduced when their cellular binding proteins or target mRNAs are artificially overexpressed (Gao and Wu, 2015).

NcRNA can also be found outside of EVs in all body fluids (Tzimagiorgis et al., 2011) and their composition differs significantly between EVs and the non-EV secretome, with
miRNAs composing the largest fraction of ncRNA found in both compartments (Langevin et al., 2020). Secretion of unbound ncRNA seems to be associated with necrosis and apoptosis of the releasing cell. Since EV-unbound DNA and RNA is similarly fragmented as the DNA/RNA in apoptotic bodies, unbound DNA/RNA could also originate from apoptotic bodies (Halicka et al., 2000; Li et al., 2003).

EV uptake into target cells is mediated through a variety of pathways (Maas et al., 2017). EVs can interact with their target cells via specific ligand-receptor interactions such as clathrinmediated endocytosis and activate downstream signaling pathways (Mulcahy et al., 2014; Costa-Silva et al., 2015). In order for ncRNAs to carry out their cellular functions, EVs not only have to bind to their target cells but need to deliver their cargo into the cytoplasma of the cell, most likely via endocytosis (Mulcahy et al., 2014). Phagocytosis of EVs is promoted when their content is lipid-rich and the extracellular environment is acidic (Parolini et al., 2009; Mulcahy et al., 2014). To prevent degradation via lysosomal fusion, ncRNAs need to escape this compartment before fusion (Stalder et al., 2013). Uptake of EV-cargo into the endosome is regarded as a potential escape mechanism for ncRNA degradation (Maas et al., 2017). NcRNAs may avoid degradation by binding to Ago and interact with the RNA interference silencing complex (RISC), a multi-protein complex at the ER that uses ncRNA as a template to cleave the corresponding mRNA (Pratt and MacRae, 2009; Stalder et al., 2013). EVs were found to encircle the ER before fusing with lysosomes, thus potentially allowing RISC and ncRNA interaction, induction of miRNA/mRNA degradation and silencing protein translation, rather than sole miRNA degradation (Barman and Bhattacharyya, 2015). 
Under ischemic conditions, cardiomyocytes transmit EVs promoting inflammation via IL6 and CC2 release. In contrast, endothelial cells can prevent cardiomyocyte apoptosis in ischemia via EV secretion and miR-transfer (Davidson et al., 2018; Loyer et al., 2018). In calcific aortic valve disease (CAVD) EVs and ncRNA expression appear to regulate the initial inflammatory phase and may promote calcification of the valve (Hutcheson et al., 2014; Bakhshian Nik et al., 2017).

In summary, ncRNA mediate a variety of cardiac diseases through uptake into endothelial cells, smooth muscle cells, cardiomyocytes as well as immune cells.

\section{THE NON-CODING TRANSCRIPTOME AS BIOMARKER: CHALLENGES AND FUTURE DIRECTIONS}

NcRNAs have become of great interest as biomarkers of various diseases (Busch et al., 2016). Circulating EV- and protein-bound ncRNAs have been shown to be differentially expressed in patients with and without $\mathrm{CV}$ diseases such as atherosclerosis, aortic aneurysms, aortic valve stenosis, and (pulmonary) hypertension (Liu et al., 2019). Levels of ncRNAs can be either increased or decreased and correlate with disease outcome, thus demonstrating their ability to serve as biomarkers of CV disease.

NcRNAs have been tested as screening biomarkers for several cardiovascular diseases such as myocardial infarction (MI), coronary artery disease $(\mathrm{CAD})$ and heart failure. In myocardial infarction miRNA-1, -133a/b, -208a, -499 became a frequently studied group referred to as myomirs due to cardiac specific interactions with different myosin chains and quick expression response after myocardial injury (van Rooij et al., 2009; Busch et al., 2016). In a study determining the correlation of miRNAs with myocardial infarction, only the levels of miRNA134 and miR-184 appeared to correlate with infarction, with miRNA-134 promoting proliferation of cardiac progenitor cells in vitro (Wu et al., 2015; Busch et al., 2016). Troponin assays were superior to circulating miRNAs in predicting myocardial infarction in patients presenting with chest pain, but some miRNAs (miR-208b) showed a high predictive value for the lifetime risk of MI (Zampetaki et al., 2012; Devaux et al., 2015).

In CAD miRNA-133a and miRNA-499 showed a positive correlation with vessel calcification, while miRNA-145 and -155 expression showed an inverse correlation with CAD-severity and progression (Busch et al., 2016). However, in a clinical setting no ncRNA was able to predict angina pectoris. The number of patients enrolled in this study was limited and, with miRNA155 shown to alter atherosclerosis in vivo in a mouse model of atherosclerosis, larger cohorts may yield more reliable results in the future (Bhattachariya et al., 2015).

Research in lncRNA as biomarkers are less advanced due to difficulties in maintaining their structural integrity in bodily fluids over prolonged periods of time (Shi and Yang, 2016). Circulating levels of the ncRNA LIPCAR were found to be upregulated in heart failure patients and could predict cardiac remodeling in general with high LIPCAR levels associated with increased cardiac mortality
(Kumarswamy et al., 2014). Vausort et al. identified three circulating lncRNAs (aHIF, KCNQ1OT1, and MALAT1) upregulated and one downregulated (ANRIL) in patients with myocardial infarction, but again all lncRNAs were inferior in predicting $\mathrm{MI}$ than conventional troponin assays (Vausort et al., 2014).

To analyze and quantify ncRNAs in exosomes and microvesicles, they must be isolated from platelet-depleted plasma (Liu et al., 2020). Isolation techniques differ between ncRNAs transported in EVs and those transported in ncRNAprotein complexes (Liu et al., 2020). A commonly used technique is differential centrifugation, although a variety of isolation methods are applied (Witwer et al., 2013). After purification of EVs, ncRNA isolation can be performed with phenol-containing reagents or phenol free assays (E et al., 2018). NcRNAs are then further analyzed using reverse transcription and quantitative PCR. Yield and purity differ immensely between different approaches as well as between research groups (E et al., 2018), and the low concentration of protein-ncRNA complexes can make quantification difficult (Gallo et al., 2012). While differential ultracentrifugation is an established method, it is time consuming and demands large sample sizes, while only producing a low recovery rate, thus making it impractical in large scale clinical settings (Liu et al., 2020). Other techniques, better suited for small sample sizes, such as spectrofluorimetry and capillary electrophoresis are more expensive and even more time consuming, and thus also not feasible in a high throughput diagnostic setting (E et al., 2018; Gallo et al., 2012). Cheaper and faster alternatives are size based methods such as ultrafiltration or hydrostatic filtration dialysis with commercial EV filter kits already established. Exosomes can also be isolated using a weight or size specific filter. However, increased mechanical sheer may break vesicles and influence results. With exosomes derived from the endocytic pathway and microvesicles formed from the plasma membrane, they express different CD-markers, making immunocapture-assays another potential route for isolation (Liu et al., 2020).

A general problem when using extracellular ncRNAs as potential biomarkers is their high dependency on sex, ethnicity and pre-analytical variabilities, as well as their altered concentrations after heparin, acetylsalicylic acid, or statin administration, making the definition of pathological threshold levels difficult (Moldovan et al., 2014; Viereck and Thum, 2017). While no international standards have been set, due to potential heparin interference blood serum obtained in the morning hours from fasting patients promises more reliable results. In order to harmonize standards a compendium of exosomal proteins and ncRNA by the International Society of Extracellular Vesicles (ISEV) has been established (Keerthikumar et al., 2016).

\section{NCRNAS AS POTENTIAL THERAPEUTIC TARGETS}

Several pathomechanisms in cardiovascular disease are influenced by ncRNA, making them promising and desirable 
targets to influence by way of ncRNA mimics or inhibitors. NcRNA therapeutics do not induce drug resistance effects in target cells and can be modified to increase their half-life, making them almost ideal therapeutic molecules (Geary et al., 2015).

In order to modulate miR- or lncRNA concentration in cardiovascular and valvular disease, RNA-based therapeutics need to reach either target cells (e.g., valvular or vascular endothelial cells, cardiomyocytes, etc.) via the blood stream or local injection (Zietzer et al., 2021). Intracardial application of RNA therapeutics demands a more invasive application pathway while i.v. or s.c. application leads to systemic distribution (Ito et al., 2009). Due to limited clinical trials, it is currently unknown how and if the local intracardial concentration of miRNA therapeutics differs between different application methods (Huang et al., 2020). In murine and porcine models, intracardial antimiR-132 concentrations in cardiomyocytes showed no difference after intravenous or intracoronary injection (Foinquinos et al., 2020).

Another hurdle for ncRNA therapeutics is identifying a transporter that can deliver its cargo specifically to its target. Virus-based approaches, using a modified adeno associated virus-capsule with an increased cardiac target specificity, are seen as a reliable transport mechanism with limited systemic effects demonstrated in rhesus macaques (Mingozzi et al., 2013). However, AAV-delivery may be limited due to potentially high adenovirus antibody titers in the general population (Calcedo et al., 2011). Also, due to its small genome size (3-4 kb capacity) the inserted ncRNA size is limited, making the overexpression of lncRNAs difficult or impossible, at least with AAV-based vectors (Smith et al., 2009). AAV transfection, when successful, leads to long-term persistence, thus pro proliferative effects must be regulated via the promoter region to avoid cancerogenic transformation (Braga et al., 2021).

Alternatively, delivery methods based on EVs and liposomes are already being tested in clinical settings (Braga et al., 2021). So called lipoplexes consist of small lipid molecules and have successfully been used to transfect cardiomyocytes in vitro and in vivo in different animals and are currently tested in clinical trials (Kulkarni et al., 2018). For systemic application it is important that lipoplexes are not positively charged to avoid increased plasma clearance as well as systemic inflammation and must not be too large $(<1 \mu \mathrm{m})$ to avoid systemic inflammatory responses and toxicity. Only recently, smaller $(<100 \mathrm{~nm})$ and ionizable or neutral lipid nanoparticles were introduced to avoid inflammation and toxicity (Kulkarni et al., 2018). For amyloidosis Patisiran is already used in a clinical setting (Hoy, 2018). Modified exosomes, through engineering of specific ligands onto the exosomal membrane, have also been tested as ncRNA transporters as well (Mathiyalagan and Sahoo, 2017). While exosome-based therapy is still in its infancy, the use of cellular organelles promises low systemic toxicity and antigenicity (Mathiyalagan and Sahoo, 2017; Braga et al., 2021). For example, trial NCT04327635 investigates patient safety in intracoronary exosomes application after myocardial infarction, which may limit systemic toxicity (McLeod, 2021).
Synthetic Nanoparticles with diameters ranging between 50 and $100 \mathrm{~nm}$ showed an optimized distribution of RNA therapeutics in vivo as well as an increased half-life (Boca et al., 2020). Their surface can easily be modified with aptamers, antibodies or peptides, potentially making them tissue specific and reducing off-target side effects (Di Mauro et al., 2018). However, their cargo capacity is limited and their effectiveness in cardiovascular cells still needs to be demonstrated (Di Mauro et al., 2018). While ncRNAs are promising targets for pharmaceutical therapy and ncRNA derived pharmaceuticals may yield almost ideal pharmacokinetic properties, their delivery method as well as their method of transportation within the body still pose major challenges.

\section{CONCLUSION}

NcRNAs are important mediators in cardiovascular and valvular disease. NcRNA expression is altered according to the parental cells' patho/physiological condition. Some ncRNAs mediate are involved in cardiovascular and valvular disease progression, while others may serve as biomarkers. EVs, lipoplexes or proteins play a key role for ncRNA transportation. Defining pathological thresholds for ncRNAs remains non-unified with ncRNA isolation techniques varying internationally. While ncRNA-based therapeutics may significantly alter cardiovascular and valvular disease progression, neither the application method nor the mode of transportation has been reliably established and this will be an important focus of future research.

\section{AUTHOR CONTRIBUTIONS}

All authors listed have made a substantial, direct, and intellectual contribution to the work and approved it for publication.

\section{FUNDING}

Open Access funding enabled and organized by Projekt DEAL. This work was supported by the medical faculty of the University of Bonn (BONFOR Grant No. 2018-1A-07 to AZ, SCIMED Grant: 2018-4-03 to PJ), by the German Cardiac Society (DGK16/2018 to AZ), the Ernst and Berta Grimmke Foundation (13/19 to AZ), the Deutsche Forschungsgemeinschaft (WE 4139/8-1 to NW, JA 2351/ 2-1 to FJ, and Project-ID 397484323-TRR 259 to FJ) and the Corona-Foundation (to FJ). NM and AP thank the DFG (397484323/SFB TRR 259/1).

\section{ACKNOWLEDGMENTS}

We thank Dr. Meghan Lucas for critical proofreading of this manuscript. 


\section{REFERENCES}

Alexopoulos, N., and Raggi, P. (2009). Calcification in atherosclerosis. Nat Rev Cardiol 6 (11), 681-688. doi:10.1038/nrcardio.2009.165

Bakhshian Nik, A., Hutcheson, J. D., and Aikawa, E. (2017). Extracellular vesicles as mediators of cardiovascular calcification. Front. Cardiovasc. Med. 4, 78. doi: $10.3389 /$ fcvm.2017.00078

Barman, B., and Bhattacharyya, S. N. (2015). mRNA targeting to endoplasmic reticulum precedes ago protein interaction and microRNA (miRNA)-mediated translation repression in mammalian cells. J. Biol. Chem. 290 (41), 24650-24656. doi:10.1074/jbc.c115.661868

Bhattachariya, A., Dahan, D., Ekman, M., Boettger, T., Braun, T., Swärd, K., et al. (2015). Spontaneous activity and stretch-induced contractile differentiation are reduced in vascular smooth muscle of miR-143/145 knockout mice. Acta Physiol 215 (3), 133-143. doi:10.1111/apha.12536

Boca, S., Gulei, D., Zimta, A.-A., Onaciu, A., Magdo, L., Tigu, A. B., et al. (2020). Nanoscale delivery systems for microRNAs in cancer therapy. Cell. Mol. Life Sci. 77 (6), 1059-1086. doi:10.1007/s00018-019-03317-9

Braga, L., Ali, H., Secco, I., and Giacca, M. (2021). Non-coding RNA therapeutics for cardiac regeneration. Cardiovasc. Res. 117 (3), 674-693. doi:10.1093/cvr/ cvaa071

Busch, A., Eken, S. M., and Maegdefessel, L. (2016). Prospective and therapeutic screening value of non-coding RNA as biomarkers in cardiovascular disease. Ann Transl Med 4 (12), 236. doi:10.21037/atm.2016.06.06

Calcedo, R., Morizono, H., Wang, L., McCarter, R., He, J., Jones, D., et al. (2011). Adeno-associated virus antibody profiles in newborns, children, and adolescents. Clin. Vaccin. Immunol. 18 (9), 1586-1588. doi:10.1128/ cvi.05107-11

Carpenter, S., Aiello, D., Atianand, M. K., Ricci, E. P., Gandhi, P., Hall, L. L., et al. (2013). A long noncoding RNA mediates both activation and repression of immune response genes. science 341 (6147), 789-792. doi:10.1126/ science. 1240925

Chong, A. J., Shimamoto, A., Hampton, C. R., Takayama, H., Spring, D. J., Rothnie, C. L., et al. (2004). Toll-like receptor 4 mediates ischemia/reperfusion injury of the heart. J. Thorac. Cardiovasc. Surg. 128 (2), 170-179. doi:10.1016/ j.jtcrs.2003.11.036

Coffey, S., and Jones, G. T. (2014). MicroRNAs are central to osteogenesis: a review with a focus on cardiovascular calcification. microRNA Diagn. Ther. 1, 1.

Cosentino, F. (2019). ESC Guidelines on diabetes, pre-diabetes, and cardiovascular diseases developed in collaboration with the EASD: The Task Force for diabetes, pre-diabetes, and cardiovascular diseases of the European Society of Cardiology (ESC) and the European Association for the Study of Diabetes (EASD). Eur. Heart J. 41 (2), 255-323.

Costa-Silva, B., Aiello, N. M., Ocean, A. J., Singh, S., Zhang, H., Thakur, B. K., et al. (2015). Pancreatic cancer exosomes initiate pre-metastatic niche formation in the liver. Nat Cel Biol 17 (6), 816-826. doi:10.1038/ncb3169

Das, S. (2020). Noncoding RNAs in cardiovascular disease: current knowledge, tools and technologies for investigation, and future directions: a scientific statement from the american heart association. Circ. Genomic Precision Med. 13 (4), e000062. doi:10.1161/hcg.0000000000000062

Davidson, S. M., Riquelme, J. A., Zheng, Y., Vicencio, J. M., Lavandero, S., and Yellon, D. M. (2018). Endothelial cells release cardioprotective exosomes that may contribute to ischaemic preconditioning. Sci Rep 8 (1), 15885-15889. doi:10.1038/s41598-018-34357-z

Devaux, Y., Mueller, M., Haaf, P., Goretti, E., Twerenbold, R., Zangrando, J., et al. (2015). Diagnostic and prognostic value of circulating microRNAs in patients with acute chest pain. J Intern Med 277 (2), 260-271. doi:10.1111/joim.12183

Di Mauro, V., Barandalla-Sobrados, M., and Catalucci, D. (2018). The noncodingRNA landscape in cardiovascular health and disease. Non-coding RNA Res. 3 (1), 12-19. doi:10.1016/j.ncrna.2018.02.001

E, S., Costa, M. C., Kurc, S., Drożdż, A., Cortez-Dias, N., and Enguita, F. J. (2018). The circulating non-coding RNA landscape for biomarker research: lessons and prospects from cardiovascular diseases. Acta Pharmacol Sin 39 (7), 1085-1099. doi:10.1038/aps.2018.35

Eguchi, T., Watanabe, K., Hara, E. S., Ono, M., Kuboki, T., and Calderwood, S. K. (2013). OstemiR: a novel panel of microRNA biomarkers in osteoblastic and osteocytic differentiation from mesencymal stem cells. PloS one 8 (3), e58796. doi:10.1371/journal.pone.0058796

Foinquinos, A., Batkai, S., Genschel, C., Viereck, J., Rump, S., Gyöngyösi, M., et al. (2020). Preclinical development of a miR-132 inhibitor for heart failure treatment. Nat Commun 11 (1), 633-710. doi:10.1038/s41467-020-14349-2

Gallo, A., Tandon, M., Alevizos, I., and Illei, G. G. (2012). The majority of microRNAs detectable in serum and saliva is concentrated in exosomes. PloS one 7 (3), e30679. doi:10.1371/journal.pone.0030679

Gao, Y.-c., and Wu, J. (2015). MicroRNA-200c and microRNA-141 as potential diagnostic and prognostic biomarkers for ovarian cancer. Tumor Biol. 36 (6), 4843-4850. doi:10.1007/s13277-015-3138-3

Geary, R. S., Norris, D., Yu, R., and Bennett, C. F. (2015). Pharmacokinetics, biodistribution and cell uptake of antisense oligonucleotides. Adv. Drug Deliv. Rev. 87, 46-51. doi:10.1016/j.addr.2015.01.008

Gezer, U., Özgür, E., Cetinkaya, M., Isin, M., and Dalay, N. (2014). Long noncoding RNAs with low expression levels in cells are enriched in secreted exosomes. Cell Biol Int 38 (9), 1076-1079. doi:10.1002/cbin.10301

Goettsch, C., Rauner, M., Pacyna, N., Hempel, U., Bornstein, S. R., and Hofbauer, L. C. (2011). miR-125b regulates calcification of vascular smooth muscle cells. Am. J. Pathol. 179 (4), 1594-1600. doi:10.1016/j.ajpath.2011.06.016

Goody, P. R., Hosen, M. R., Christmann, D., Niepmann, S. T., Zietzer, A., Adam, M., et al. (2020). Aortic Valve Stenosis. Atvb 40 (4), 885-900. doi:10.1161/ atvbaha.119.313067

Guttman, M., Amit, I., Garber, M., French, C., Lin, M. F., Feldser, D., et al. (2009). Chromatin signature reveals over a thousand highly conserved large noncoding RNAs in mammals. Nature 458 (7235), 223-227. doi:10.1038/ nature 07672

Halicka, H. D., Bedner, E., and Darzynkiewicz, Z. (2000). Segregation of RNA and separate packaging of DNA and RNA in apoptotic bodies during apoptosis. Exp. Cel. Res. 260 (2), 248-256. doi:10.1006/excr.2000.5027

Hoy, S. M. (2018). Patisiran: first global approval. Drugs 78 (15), 1625-1631. doi:10.1007/s40265-018-0983-6

Huang, C.-K., Kafert-Kasting, S., and Thum, T. (2020). Preclinical and clinical development of noncoding RNA therapeutics for cardiovascular disease. Circ Res 126 (5), 663-678. doi:10.1161/circresaha.119.315856

Hutcheson, J. D., Maldonado, N., and Aikawa, E. (2014). Small entities with large impact. Curr. Opin. Lipidol. 25 (5), 327-332. doi:10.1097/ mol.0000000000000105

Ito, Y., Kawakami, S., Charoensit, P., Higuchi, Y., and Hashida, M. (2009). Evaluation of proinflammatory cytokine production and liver injury induced by plasmid DNA/cationic liposome complexes with various mixing ratios in mice. Eur. J. pharmaceutics biopharmaceutics 71 (2), 303-309. doi:10.1016/ j.ejpb.2008.09.005

Kattoor, A. J., Pothineni, N. V. K., Palagiri, D., and Mehta, J. L. (2017). Oxidative stress in atherosclerosis. Curr Atheroscler Rep 19 (11), 42-11. doi:10.1007/ s11883-017-0678-6

Keerthikumar, S., Chisanga, D., Ariyaratne, D., Al Saffar, H., Anand, S., Zhao, K., et al. (2016). ExoCarta: a web-based compendium of exosomal cargo. J. Mol. Biol. 428 (4), 688-692. doi:10.1016/j.jmb.2015.09.019

Kim, K. M., Abdelmohsen, K., Mustapic, M., Kapogiannis, D., and Gorospe, M. (2017). RNA in extracellular vesicles. WIREs RNA 8 (4), e1413. doi:10.1002/ wrna. 1413

Kulkarni, J. A., Cullis, P. R., and Van Der Meel, R. (2018). Lipid nanoparticles enabling gene therapies: from concepts to clinical utility. Nucleic acid Ther. 28 (3), 146-157. doi:10.1089/nat.2018.0721

Kumarswamy, R., Bauters, C., Volkmann, I., Maury, F., Fetisch, J., Holzmann, A., et al. (2014). Circulating long noncoding RNA, LIPCAR, predicts survival in patients with heart failure. Circ Res 114 (10), 1569-1575. doi:10.1161/ circresaha.114.303915

Langevin, S. M., Kuhnell, D., Biesiada, J., Zhang, X., Medvedovic, M., Talaska, G. G., et al. (2020). Comparability of the small RNA secretome across human biofluids concomitantly collected from healthy adults. PloS one 15 (4), e0229976. doi:10.1371/journal.pone.0229976

Li, C.-N., Hsu, H.-L., Wu, T.-L., Tsao, K.-C., Sun, C.-F., and Wu, J. T. (2003). Cell-free DNA is released from tumor cells upon cell death: A study of tissue cultures of tumor cell lines. J. Clin. Lab. Anal. 17 (4), 103-107. doi:10.1002/ jcla. 10081 
Libby, P., Buring, J. E., Badimon, L., Hansson, G. K., Deanfield, J., Bittencourt, M. S., et al. (2019). Atherosclerosis. Nat Rev Dis Primers 5 (1), 56. doi:10.1038/ s41572-019-0106-z

Liu, C., Wu, H.-T., Zhu, N., Shi, Y.-N., Liu, Z., Ao, B.-X., et al. (2016). Steroid receptor RNA activator: Biologic function and role in disease. Clinica Chim. Acta 459, 137-146. doi:10.1016/j.cca.2016.06.004

Liu, J., Wu, J., Li, L., Li, T., and Wang, J. (2020). The Role of Exosomal Non-Coding RNAs in Coronary Artery Disease. Front. Pharmacol., 11. doi:10.3389/ fphar.2020.603104

Liu, S., Sheng, L., Miao, H., Saunders, T. L., MacDougald, O. A., Koenig, R. J., et al. (2014). SRA gene knockout protects against diet-induced obesity and improves glucose tolerance. J. Biol. Chem. 289 (19), 13000-13009. doi:10.1074/ jbc.m114.564658

Liu, S., Xu, R., Gerin, I., Cawthorn, W. P., MacDougald, O. A., Chen, X.-W., et al. (2014). SRA regulates adipogenesis by modulating p38/JNK phosphorylation and stimulating insulin receptor gene expression and downstream signaling. PloS one 9 (4), e95416. doi:10.1371/journal.pone.0095416

Liu, Y., Ferguson, J. F., Xue, C., Ballantyne, R. L., Silverman, I. M., Gosai, S. J., et al. (2014). Tissue-specific RNA-Seq in human evoked inflammation identifies blood and adipose LincRNA signatures of cardiometabolic diseases. Atvb 34 (4), 902-912. doi:10.1161/atvbaha.113.303123

Liu, Y., Li, Q., Hosen, M. R., Zietzer, A., Flender, A., Levermann, P., et al. (2019). Atherosclerotic conditions promote the packaging of functional microRNA92a-3p into endothelial microvesicles. Circ Res 124 (4), 575-587. doi:10.1161/ circresaha.118.314010

Loyer, X., Zlatanova, I., Devue, C., Yin, M., Howangyin, K.-Y., Klaihmon, P., et al. (2018). Intra-cardiac release of extracellular vesicles shapes inflammation following myocardial infarction. Circ Res 123 (1), 100-106. doi:10.1161/ circresaha.117.311326

Maas, S. L. N., Breakefield, X. O., and Weaver, A. M. (2017). Extracellular vesicles: unique intercellular delivery vehicles. Trends Cell Biology 27 (3), 172-188. doi:10.1016/j.tcb.2016.11.003

Mahmut, A., Boulanger, M.-C., El Husseini, D., Fournier, D., Bouchareb, R., Després, J.-P., et al. (2014). Elevated Expression of Lipoprotein-Associated Phospholipase A2 in Calcific Aortic Valve Disease. J. Am. Coll. Cardiol. 63 (5), 460-469. doi:10.1016/j.jacc.2013.05.105

Mathiyalagan, P., and Sahoo, S. (2017). "Exosomes-based gene therapy for microRNA delivery," in Cardiac Gene Therapy (Berlin: Springer), 139-152. doi:10.1007/978-1-4939-6588-5_9

McLeod, C. J. (2021). Safety Evaluation of Intracoronary Infusion of Extracellular Vesicles in Patients with AMI.

Mingozzi, F., Anguela, X. M., Pavani, G., Chen, Y., Davidson, R. J., Hui, D. J., et al. (2013). Overcoming preexisting humoral immunity to AAV using capsid decoys. Sci. translational Med. 5 (194), 194ra92. doi:10.1126/ scitranslmed.3005795

Moldovan, L., Batte, K. E., Trgovcich, J., Wisler, J., Marsh, C. B., and Piper, M. (2014). Methodological challenges in utilizing mi RNA $s$ as circulating biomarkers. J. Cel. Mol. Med. 18 (3), 371-390. doi:10.1111/ jcmm. 12236

Mulcahy, L. A., Pink, R. C., and Carter, D. R. F. (2014). Routes and mechanisms of extracellular vesicle uptake. J. extracellular vesicles 3 (1), 24641. doi:10.3402/ jev.v3.24641

Nigam, V., Sievers, H. H., Jensen, B. C., Sier, H. A., Simpson, P. C., Srivastava, D., et al. (2010). Altered microRNAs in bicuspid aortic valve: a comparison between stenotic and insufficient valves. J Heart Valve Dis 19 (4), 459-465.

Parolini, I., Federici, C., Raggi, C., Lugini, L., Palleschi, S., De Milito, A., et al. (2009). Microenvironmental $\mathrm{pH}$ is a key factor for exosome traffic in tumor cells. J. Biol. Chem. 284 (49), 34211-34222. doi:10.1074/jbc.m109.041152

Pratt, A. J., and MacRae, I. J. (2009). The RNA-induced silencing complex: a versatile gene-silencing machine. J. Biol. Chem. 284 (27), 17897-17901. doi:10.1074/jbc.r900012200

Radhakrishnan, R., and Kowluru, R. A. (2021). Long noncoding RNA MALAT1 and regulation of the antioxidant defense system in diabetic retinopathy. Diabetes 70 (1), 227-239. doi:10.2337/db20-0375

Rangrez, A. Y., M'Baya-Moutoula, E., Metzinger-Le Meuth, V., Hénaut, L., Djelouat, M. S. e. I., Benchitrit, J., et al. (2012). Inorganic phosphate accelerates the migration of vascular smooth muscle cells: evidence for the involvement of miR-223. PloS one 7 (10), e47807. doi:10.1371/ journal.pone.0047807

Rapicavoli, N. A., Qu, K., Zhang, J., Mikhail, M., Laberge, R. M., and Chang, H. Y. (2013). A mammalian pseudogene lncRNA at the interface of inflammation and anti-inflammatory therapeutics. elife 2, e00762. doi:10.7554/eLife.00762

Rayner, K. J. (2020). Leading the Long Noncoding RNA Pack: PELATON in Human Atherosclerosis. US: Am Heart Assoc.

Shi, Q., and Yang, X. (2016). Circulating microRNA and long noncoding RNA as biomarkers of cardiovascular diseases. J. Cel. Physiol 231 (4), 751-755. doi:10.1002/jcp. 25174

Shurtleff, M. J., Temoche-Diaz, M. M., Karfilis, K. V., Ri, S., and Schekman, R. (2016). Y-box protein 1 is required to sort microRNAs into exosomes in cells and in a cell-free reaction. elife 5, e19276. doi:10.7554/eLife.19276

Smith, A. C., Poulin, K. L., and Parks, R. J. (2009). DNA genome size affects the stability of the adenovirus virion. J Virol 83 (4), 2025-2028. doi:10.1128/ jvi.01644-08

Song, R., Zhai, Y., Ao, L., Fullerton, D. A., and Meng, X. (2020). MicroRNA-204 deficiency in human aortic valves elevates valvular osteogenic activity. Int. J. Mol. Sci. 21 (1), 76.

Stalder, L., Heusermann, W., Sokol, L., Trojer, D., Wirz, J., Hean, J., et al. (2013). The rough endoplasmatic reticulum is a central nucleation site of siRNAmediated RNA silencing. Embo J 32 (8), 1115-1127. doi:10.1038/emboj.2013.52

Sun, X., and Wong, D. (2016). Long non-coding RNA-mediated regulation of glucose homeostasis and diabetes. Am J Cardiovasc Dis 6 (2), 17-25.

Tzimagiorgis, G., Michailidou, E. Z., Kritis, A., Markopoulos, A. K., and Kouidou, S. (2011). Recovering circulating extracellular or cell-free RNA from bodily fluids. Cancer Epidemiol. 35 (6), 580-589. doi:10.1016/j.canep.2011.02.016

van Rooij, E., Quiat, D., Johnson, B. A., Sutherland, L. B., Qi, X., Richardson, J. A., et al. (2009). A family of microRNAs encoded by myosin genes governs myosin expression and muscle performance. Dev. Cel. 17 (5), 662-673. doi:10.1016/ j.devcel.2009.10.013

Vausort, M., Wagner, D. R., and Devaux, Y. (2014). Long noncoding RNAs in patients with acute myocardial infarction. Circ Res 115 (7), 668-677. doi:10.1161/circresaha.115.303836

Venardos, N., Nadlonek, N. A., Zhan, Q., Weyant, M. J., Reece, T. B., Meng, X., et al. (2014). Aortic valve calcification is mediated by a differential response of aortic valve interstitial cells to inflammation. J. Surg. Res. 190 (1), 1-8. doi:10.1016/j.jss.2014.03.051

Viereck, J., and Thum, T. (2017). Circulating noncoding RNAs as biomarkers of cardiovascular disease and injury. Circ Res 120 (2), 381-399. doi:10.1161/ circresaha.116.308434

Villarroya-Beltri, C., Gutiérrez-Vázquez, C., Sánchez-Cabo, F., Pérez-Hernández, D., Vázquez, J., Martin-Cofreces, N., et al. (2013). Sumoylated hnRNPA2B1 controls the sorting of miRNAs into exosomes through binding to specific motifs. Nat Commun 4 (1), 2980-3010. doi:10.1038/ncomms3980

Wang, J., Guan, X., Guo, F., Zhou, J., Chang, A., Sun, B., et al. (2013). miR-30e reciprocally regulates the differentiation of adipocytes and osteoblasts by directly targeting low-density lipoprotein receptor-related protein 6. Cell Death Dis 4 (10), e845. doi:10.1038/cddis.2013.356

Wang, Y., Xiao, X., Zhou, T., Han, D., and Dong, N. (2020). Novel mechanisms for osteogenic differentiation of human aortic valve interstitial cells. J. Thorac. Cardiovasc. Surg. 159 (5), 1742-1753. e7. doi:10.1016/j.jtcvs.2019.05.051

Whitley, M. Z., Williams, A. J., and Collins, T. (1994). NF-kappa B and I kappa B alpha: an inducible regulatory system in endothelial activation. J. Exp. Med. 179 (2), 503-512.

Witwer, K. W., Buzás, E. I., Bemis, L. T., Bora, A., Lässer, C., Lötvall, J., et al. (2013). Standardization of sample collection, isolation and analysis methods in extracellular vesicle research. J. extracellular vesicles 2 (1), 20360. doi:10.3402/jev.v2i0.20360

Wu, Y.-H., Zhao, H., Zhou, L.-P., Zhao, C.-X., Wu, Y.-F., Zhen, L.-X., et al. (2015). miR-134 modulates the proliferation of human cardiomyocyte progenitor cells by targeting Meis2. Ijms 16 (10), 25199-25213. doi:10.3390/ijms161025199

Xu, B., Gerin, I., Miao, H., Vu-Phan, D., Johnson, C. N., Xu, R., et al. (2010). Multiple roles for the non-coding RNA SRA in regulation of adipogenesis and insulin sensitivity. PloS one 5 (12), e14199. doi:10.1371/journal.pone.0014199

Xu, E., Hu, X., Li, X., Jin, G., Zhuang, L., Wang, Q., et al. (2020). Analysis of long non-coding RNA expression profiles in high-glucose treated vascular 
endothelial cells. BMC Endocr Disord 20 (1), 107-110. doi:10.1186/s12902-02000593-6

Xu, J.-J., Zheng, W.-H., Wang, J., and Chen, Y.-Y. (2020). Long non-coding RNA plasmacytoma variant translocation 1 linked to hypoxia-induced cardiomyocyte injury of H9c2 cells by targeting miR-135a-5p/forkhead box O1 axis. Chin. Med. J. 133 (24), 2953-2962. doi:10.1097/ cm9.0000000000001147

Yan, B., Yao, J., Liu, J.-Y., Li, X.-M., Wang, X.-Q., Li, Y.-J., et al. (2015). IncRNA-MIAT regulates microvascular dysfunction by functioning as a competing endogenous RNA. Circ Res 116 (7), 1143-1156. doi:10.1161/circresaha.116.305510

Yuan, T., Yang, T., Chen, H., Fu, D., Hu, Y., Wang, J., et al. (2019). New insights into oxidative stress and inflammation during diabetes mellitus-accelerated atherosclerosis. Redox Biol. 20, 247-260. doi:10.1016/j.redox.2018.09.025

Zampetaki, A., Willeit, P., Tilling, L., Drozdov, I., Prokopi, M., Renard, J.-M., et al. (2012). Prospective study on circulating MicroRNAs and risk of myocardial infarction. J. Am. Coll. Cardiol. 60 (4), 290-299. doi:10.1016/j.jacc.2012.03.056

Zietzer, A., Hosen, M. R., Wang, H., Goody, P. R., Sylvester, M., Latz, E., et al. (2020). The RNA-binding protein hnRNPU regulates the sorting of microRNA30c-5p into large extracellular vesicles. J. extracellular vesicles 9 (1), 1786967. doi:10.1080/20013078.2020.1786967
Zietzer, A., Nickenig, G., and Jansen, F. (2021). Small blebs, big potential-can extracellular vesicles cure cardiovascular disease. Eur. Heart J.

Conflict of Interest: The authors declare that the research was conducted in the absence of any commercial or financial relationships that could be construed as a potential conflict of interest.

Publisher's Note: All claims expressed in this article are solely those of the authors and do not necessarily represent those of their affiliated organizations, or those of the publisher, the editors and the reviewers. Any product that may be evaluated in this article, or claim that may be made by its manufacturer, is not guaranteed or endorsed by the publisher.

Copyright (๔ 2021 Bartsch, Goody, Hosen, Nehl, Mohammadi, Zietzer, Düsing, Pfeifer, Nickenig and Jansen. This is an open-access article distributed under the terms of the Creative Commons Attribution License (CC BY). The use, distribution or reproduction in other forums is permitted, provided the original author(s) and the copyright owner(s) are credited and that the original publication in this journal is cited, in accordance with accepted academic practice. No use, distribution or reproduction is permitted which does not comply with these terms. 